\title{
Concentración plasmática de zinc en vacas de cría de la cuenca deprimida del río Salado
}

\section{Plasma zinc concentrations in beef cows at Salado River Basin}

\author{
Galarza, Esteban Martín; Lizarraga, Raúl Martín; Mattioli, Guillermo \\ Alberto; Anchordoquy, Juan Mateo; Fazzio, Luis Emilio
}

\section{Esteban Martín Galarza}

Cátedra de Fisiología, Laboratorio de Nutrición

Mineral. Facultad de Ciencias Veterinarias, Universidad Nacional de La Plata, Argentina mgalarzaestebanm@gmail.com

\section{Raúl Martín Lizarraga}

Laboratorio de Nutrición Mineral. Facultad de Ciencias Veterinarias, Universidad Nacional de La Plata. Argentina

\section{Guillermo Alberto Mattioli}

Cátedra de Fisiología, Laboratorio de Nutrición Mineral. Facultad de Ciencias Veterinarias, Universidad Nacional de La Plata, Argentina

\section{(i) Juan Mateo Anchordoquy}

Cátedra de Fisiología, Instituto de Genética Veterinaria (IGEVET-CONICET).Facultad de Ciencias Veterinarias, Universidad Nacional de La Plata, Argentina

\section{(iD) Luis Emilio Fazzio}

Laboratorio de Nutrición Mineral, Laboratorio de Patología Especial Veterinaria(LAPEVET). Facultad de Ciencias Veterinarias, Universidad Nacional de La Plata, Argentina

\section{ANALECTA VETERINARIA \\ Universidad Nacional de La Plata, Argentina \\ ISSN: 1514-2590 \\ Periodicidad: Semestral \\ vol.41,núm.2,e057,2021 \\ analecta@fcv.unlp.edu.ar}

Recepción: 04 Mayo 2021

Revisado: 25 Mayo 2021

Aprobación: 18 Agosto 2021

URL:

http://portal.amelica.org/ameli/jatsRepo/25/25244000 $1 /$ index.html

DOI: https://doi.org/doi.org/10.24215/15142590e057
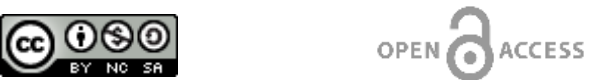

Resumen: El zinc ( $\mathrm{Zn}$ ) es un micromineral esencial y su carencia puede afectar la eficiencia productiva y reproductiva del ganado. Con el objetivo de evaluar el estatus de $\mathrm{Zn}$ en vacas de cría de la cuenca deprimida del río Salado (centro este de la provincia de Buenos Aires, Argentina), se realizó un estudio observacional multicéntrico en 110 rebaños en el que se recolectaron muestras de sangre $(\mathrm{n}=1.078$ vacas) y se analizaron mediante espectrofotometría de absorción atómica. Las vacas se clasificaron según la concentración plasmática de Zn en fisiológicamente adecuadas $\geq 90 \mu \mathrm{g} / \mathrm{dl}$, marginales entre 80 y $89 \mu \mathrm{g} / \mathrm{dl}$ y carentes $<80 \mu \mathrm{g} / \mathrm{dl}$. Se realizó un análisis estadístico descriptivo mediante el procedimiento MEANS del programa SAS. La media de la concentración plasmática de $\mathrm{Zn}$ fue de 102,8 $\pm 16,4 \mu \mathrm{g} / \mathrm{dl}$ y el $31,4 \%$ de las vacas de cada rebaño presentó valores marginales o de carencia. Durante el invierno se registró el porcentaje más alto de vacas con concentraciones plasmáticas de $\mathrm{Zn}$ marginales o carentes (47,7\%), mientras que en otoño se registró el porcentaje más bajo (21,3\%). Los resultados obtenidos demuestran que existen valores plasmáticos marginales y de carencia de $\mathrm{Zn}$ en vacas de cría de la cuenca deprimida del río Salado.

Palabras clave: zinc, bovinos, carencia mineral.

Abstract: Zinc ( $\mathrm{Zn}$ ) is an essential micromineral and its deficiency can affect the productive and reproductive efficiency in cattle. A multicenter observational study was carried out to evaluate $\mathrm{Zn}$ status in breeding cows at Salado River Basin (Buenos Aires, Argentina). The study included 110 cow-calf operations, in which blood samples ( $n=1078$ cows) were collected and analyzed by using atomic absorption spectrophotometry. Cows were classified according to their plasma $\mathrm{Zn}$ concentration values as physiologically adequate $(\geq 90 \mu \mathrm{g} / \mathrm{dl})$, marginal (between 80 to $89 \mu \mathrm{g} / \mathrm{dl})$ and deficient $(<80$ $\mu \mathrm{g} / \mathrm{dl})$. A descriptive statistical analysis was performed using the SAS program MEANS procedure. The average plasma $\mathrm{Zn}$ concentration was $102.8 \pm 16.4 \mu \mathrm{g} / \mathrm{dl}$ and $31.4 \%$ of the cows in each herd showed marginal or deficient values. The highest percentage of marginal or deficient cows was recorded in winter $(47.7 \%)$, while the lowest percentage was recorded in autumn (21.3\%). These results show that there is $\mathrm{Zn}$ deficiency in cow-calf operations of Salado River Basin.

Keywords: zinc, cattle, mineral deficiency. 


\section{Introducción}

La producción de bovinos para carne es una de las principales actividades agropecuarias de la Provincia de Buenos Aires, Argentina. Esta provincia aporta el $34 \%$ del ganado bovino para carne del país, con más de 15,7 millones de cabezas (Rossanigo et al., 2012). La mayor densidad de bovinos de la provincia se encuentra en la región productiva denominada cuenca deprimida del río Salado (CDRS) (SENASA, 2015). Esta región es una extensa llanura anegable, debido a la poca pendiente y lento drenaje, con clima templado húmedo $\mathrm{y}$ un promedio de precipitaciones anuales próximo a los $1000 \mathrm{~mm}$ (Otondo, 2011; Rojas et al., 2011). La CDRS se encuentra en el centro este de la provincia, y si bien no tiene una delimitación rigurosamente definida, está compuesta por más de veinte municipios (Mattioli, 1998; Ministerio de Agroindustria, 2015; Némoz et al., 2013;) (Figura 1).

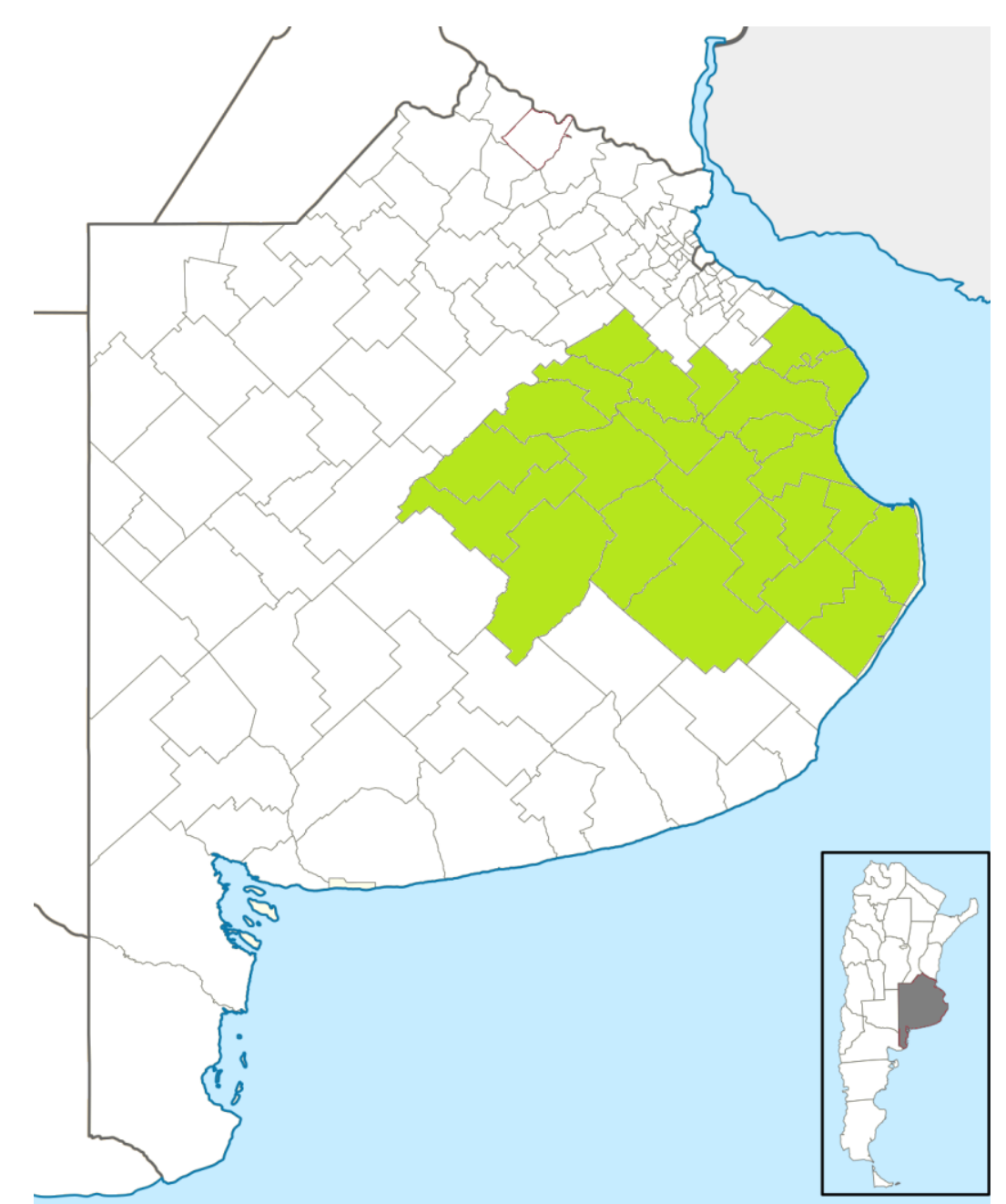

Figura 1. Municipios que componen la zona productiva denominada cuenca deprimida del río Salado. 
La producción anual de terneros en la CDRS es aproximadamente de 2 millones de cabezas, lo que representa el $34 \%$ de la existencia provincial y el $17 \%$ de la nacional; lo que la convierte en la principal zona de cría de terneros del país (Némoz et al., 2013). La producción ganadera en la región es de tipo extensiva y la alimentación de los bovinos depende en un $75 \%$ de pastizales naturales, seguido de pasturas implantadas y verdeos, con muy poca suplementación con alimentos concentrados (Faverin \& Machado, 2019; Vázquez et al., 2008). El hecho de que haya una baja implementación de suplementación condiciona a los animales a que reciban del pasto los nutrientes necesarios para un adecuado desempeño productivo y reproductivo. Dentro de estos nutrientes esenciales se encuentran los microminerales, como lo son el cobre $(\mathrm{Cu})$, el selenio, el manganeso, el iodo, el cobalto, el hierro y el zinc (Zn) (Mattioli, 2019). La concentración de microminerales en el forraje depende de muchos factores, como la concentración del mineral en el suelo, su $\mathrm{pH}$, su solubilidad, la especie forrajera y el estado fenológico de la planta, entre otros (Nicholas, 1975; Robson, 1993; Tyler \& Olsson, 2001). Si la concentración de estos minerales en el forraje es menor a la requerida por los animales, sobrevienen los cuadros de carencias (Suttle, 2010). Para el caso del $\mathrm{Zn}$, si bien no están del todo definidos los requerimientos en bovinos en pastoreo, la concentración en la pastura debe ser de, al menos, $30 \mathrm{mg} / \mathrm{kg}$ de materia seca (NRC, 2016).

El Zn es un mineral esencial ya que cumple un rol estructural, catalítico y regulatorio de la actividad celular (Levaot \& Hershfinkel, 2018). En este sentido, más de 2800 proteínas son dependientes de $\mathrm{Zn}$, entre ellas más de 300 metaloenzimas y alrededor del $10 \%$ de las proteínas asociadas a la transcripción génica (Andreini et al., 2006; Andreini \& Bertini, 2012; Tapiero \& Tew, 2003). Las enzimas dependientes de $\mathrm{Zn}$ cumplen funciones muy variadas y forman parte de la mayoría de las vías metabólicas del organismo (Kambe et al., 2015; Olechnowicz et al., 2018; Vallee \& Falchuk, 1993). Este mineral, por ejemplo, es necesario para la división y diferenciación celular, ya que participa en la síntesis y en la transcripción del ADN (Bonaventura et al., 2015; Vallee \& Falchuk, 1993). Actúa en la defensa antioxidante, debido a que forma parte de la enzima $\mathrm{Cu} / \mathrm{Zn}$ superóxido dismutasa $(\mathrm{Cu} / \mathrm{Zn}-\mathrm{SOD})$, induce la síntesis de proteínas con capacidad antioxidante como la metalotioneína y compite por los sitios de unión en las membranas biológicas con otros metales que inducen peroxidación (Lee, 2018). Por otro lado, el Zn interviene en la respiración celular y en el equilibrio ácido base ya que cumple un rol catalítico en la enzima anhidrasa carbónica (Lindskog, 1997). Por lo expuesto, es de esperar que la carencia de este mineral genere consecuencias variadas, tales como alopecia, paraqueratosis, fallas en la respuesta inmune, alteraciones en la función reproductiva y alteraciones en el crecimiento reflejadas como bajas ganancias de peso (Alsaad et al., 2006; Engle et al., 1997; Fallah et al., 2018; Ibs \& Rink, 2003; Suttle, 2010; Taneja \& Kaur, 1990).

Para determinar el estatus de $\mathrm{Zn}$ en animales se han utilizado diversos indicadores, tales como su concentración en tejidos como pelo e hígado, o la actividad sérica o plasmática de las enzimas dependientes de $\mathrm{Zn}$ como la fosfatasa alcalina; sin embargo, la concentración plasmática o sérica del mineral es el más utilizado debido a su practicidad (Herdt \& Hoff, 2011; Kincaid, 1999). Existe acuerdo en considerar fisiológicamente adecuadas a concentraciones de $\mathrm{Zn}$ en plasma por encima de $90 \mu \mathrm{g} / \mathrm{dl}$, mientras que 
valores menores a $80 \mu \mathrm{g} / \mathrm{dl}$ indicarían carencia, dejando entre ambos un rango de carencia marginal (Enjalbert et al., 2006; Mattioli, 2019). A su vez, concentraciones plasmáticas de $\mathrm{Zn}$ superiores a $300 \mu \mathrm{g} / \mathrm{dl}$ indicarían un estado de toxicidad (Kincaid, 1999). Si bien en la CDRS existen antecedentes de forrajes con concentraciones de $\mathrm{Zn}$ por debajo de lo requerido por los bovinos (Cseh et al., 1997; Ramírez et al., 1998), poco se conoce acerca del estatus de Zn en las vacas de cría de dicha región.

La hipótesis de este trabajo es que existe carencia de $\mathrm{Zn}$ en bovinos de la CDRS. Por lo tanto, el objetivo fue evaluar la concentración plasmática de $\mathrm{Zn}$ en vacas de cría, en distintos municipios que componen la CDRS.

\section{Materiales y métodos}

Las prácticas en animales fueron aprobadas bajo el protocolo para el uso de animales en investigación científica ( $\mathrm{N}^{\mathrm{O}} 5 \mathbf{1}^{-5}-15 \mathrm{~T}$ ) del Comité Institucional para el Cuidado y Uso de Animales de Laboratorio (CICUAL) de la Universidad Nacional de La Plata.

Se realizó un estudio observacional multicéntrico, entre enero de 2015 y diciembre de 2019, en 110 rebaños de cría bovina pertenecientes a 20 municipios de la CDRS. Los establecimientos seleccionados realizaban una producción de cría extensiva, sin suplementación oral de minerales. Se tomaron muestras de sangre, por una única vez, de un total de 1078 vacas (entre 7 y 12 por rebaño) con al menos un parto en su vida y clínicamente sanas al momento del muestreo. Los municipios involucrados y la distribución de los muestreos en los mismos, así como también en los años de muestreo y las estaciones del año, se presentan en la Tabla 1.

\section{Muestras de sangre}

Las muestras de sangre fueron tomadas en conjunto con los médicos veterinarios asesores de los rebaños. La extracción se realizó mediante venopunción yugular con agujas y jeringas estériles nuevas. Las muestras de sangre fueron depositadas en tubos plásticos con heparina sódica como anticoagulante. Luego de su obtención, fueron remitidas refrigeradas, dentro de las 12 horas de extraídas, al Laboratorio de Nutrición Mineral de la Facultad de Ciencias Veterinarias de la Universidad Nacional de La Plata (FCV-UNLP) o, según la cercanía, a uno de los tres laboratorios que colaboraron en este trabajo (Laboratorio Regional Rauch, Bazkos Viejos y CSG Laboratorio de Diagnóstico Veterinario, de los municipios de Rauch, General Madariaga y Lezama, respectivamente).

\section{Procesamiento de las muestras}

El primer paso (el único realizado en los laboratorios colaboradores) consistió en la separación del plasma por medio de centrifugación a 3000 rpm durante 5 minutos. Una vez obtenido, se depositó en viales plásticos que se conservaron a $-20{ }^{\circ} \mathrm{C}$ para su posterior envío, refrigerados adecuadamente, a la FCV-UNLP y allí continuar con su procesamiento. Cada muestra fue 
desproteinizada con ácido tricloracético al $10 \%(\mathrm{p} / \mathrm{v})$ y centrifugada a 5000 rpm durante 10 minutos. Con el sobrenadante obtenido se determinó la concentración plasmática de $\mathrm{Zn}$ mediante espectrofotometría de absorción atómica de llama (AAnalyst 200-Perkin Elmer, AAS, International Equipment Trading Ltd., Mundelein, USA). Para el procedimiento se utilizaron estándares internos de 0, 50, 100 y $200 \mu \mathrm{g} / \mathrm{dl}$ de $\mathrm{Zn}$.

\begin{tabular}{|c|c|c|}
\hline Municipio & $N^{o}$ de rebaños & $\mathrm{N}^{\mathrm{o}}$ de animales \\
\hline Ayacucho & 10 & 100 \\
\hline Azul & 2 & 20 \\
\hline Castelli & 4 & 41 \\
\hline Chascomús & 6 & 58 \\
\hline Dolores & 2 & 20 \\
\hline General Alvear & 8 & 82 \\
\hline General Belgrano & 2 & 17 \\
\hline General Lavalle & 10 & 99 \\
\hline General Madariaga & 11 & 110 \\
\hline General Paz & 2 & 20 \\
\hline Las Flores & 2 & 20 \\
\hline Lezama & 3 & 28 \\
\hline Lobos & 2 & 20 \\
\hline Magdalena & 2 & 20 \\
\hline Maipú & 5 & 51 \\
\hline Pila & 3 & 25 \\
\hline Punta Indio & 8 & 80 \\
\hline Rauch & 19 & 173 \\
\hline Saladillo & 4 & 42 \\
\hline Tapalqué & 5 & 52 \\
\hline \multicolumn{3}{|l|}{ Estación del año } \\
\hline Invierno & 21 & 201 \\
\hline Primavera & 13 & 129 \\
\hline Verano & 23 & 225 \\
\hline Otoño & 53 & 523 \\
\hline \multicolumn{3}{|l|}{ Año de muestreo } \\
\hline 2015 & 23 & 230 \\
\hline 2016 & 59 & 568 \\
\hline 2017 & 13 & 130 \\
\hline 2018 & 10 & 105 \\
\hline 2019 & 5 & 45 \\
\hline
\end{tabular}

Tabla 1. Distribución del número de rebaños y de animales muestreados por municipio, estación del año y año de muestreo. 


\section{Análisis estadístico}

Se realizó un análisis descriptivo de los datos con el procedimiento MEANS de SAS (SAS 9.4, Inst. Inc.). Se formaron tres grupos de vacas según sus valores de concentración plasmática de $\mathrm{Zn}$ : valores adecuados $(\geq 90 \mu \mathrm{g} / \mathrm{dl})$, marginales (entre 80 y $89 \mu \mathrm{g} / \mathrm{dl}$ ) y carentes (\# $80 \mu \mathrm{g} / \mathrm{dl}$ ) (Enjalbert et al., 2006; Mattioli, 2019). Para realizar el análisis se utilizó la media de la concentración plasmática de $\mathrm{Zn}$ del rebaño, como así también el porcentaje de animales con valores de concentración plasmática de $\mathrm{Zn}$ adecuados, marginales y carentes. Los datos se presentan en función de los municipios donde fueron tomadas las muestras, como así también el año y la estación del año en que se realizó el muestreo.

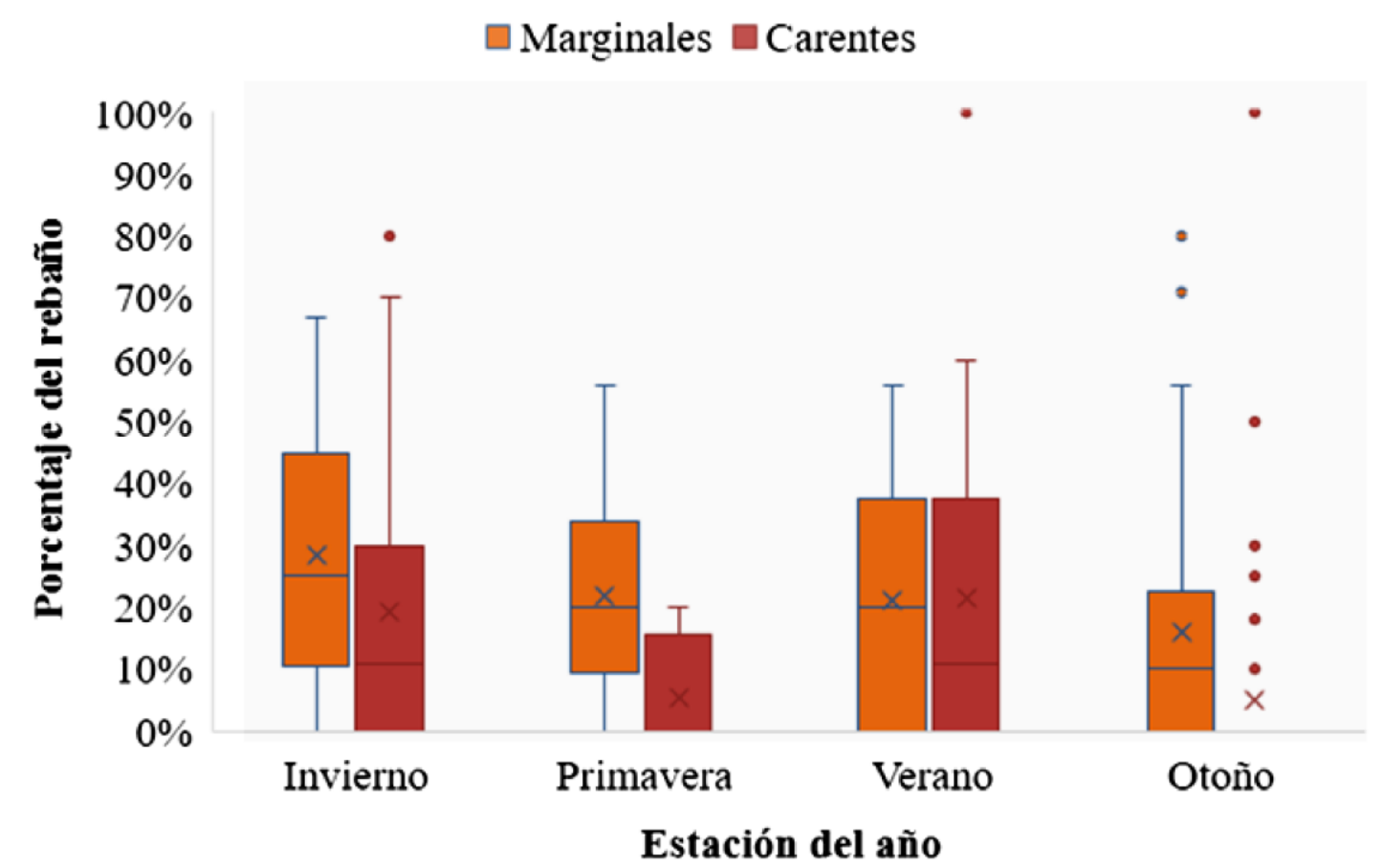

Figura 2. Porcentaje de vacas del rebaño con valores plasmáticos de Zn marginales ( 80 a $89 \mu \mathrm{gg} / \mathrm{dl}$ ) o carentes $(<80 \mu \mathrm{g} / \mathrm{dl})$ por estación del año, en la cuenca deprimida del río Salado (Buenos Aires, Argentina).

\section{Resultados}

La media de la concentración plasmática de $\mathrm{Zn}$ en los rebaños evaluados fue de 102,8 \pm 16,4 $\mu \mathrm{g} / \mathrm{dl}$, donde, en promedio, el 68,6\% ( $\pm 29,5 \%$ ) de las vacas presentaron valores plasmáticos de $\mathrm{Zn}$ adecuados, el 20,2 \% ( \pm 18,2 \%) valores marginales y el 11,2\% ( $\pm 20,4 \%)$ valores de carencia. Los valores medios registrados en invierno, primavera, verano y otoño fueron 93,2 $\pm 16,5 \mu \mathrm{g} / \mathrm{dl}$, $104,8 \pm 12,4 \mu \mathrm{g} / \mathrm{dl}, 97,4 \pm 19,3 \mu \mathrm{g} / \mathrm{dl}$ y 108,4 $\pm 13,5 \mu \mathrm{g} / \mathrm{dl}$, respectivamente. Los porcentajes de vacas del rebaño con valores plasmáticos de Zn marginales o carentes por estación se presentan en la Figura 2. Los valores medios de concentración plasmática de Zn de los rebaños en los años 2015, 2016, 2017, 2018 y 2019 fueron $103 \pm 19,4 \mu \mathrm{g} / \mathrm{dl}, 104,7 \pm 16,7 \mu \mathrm{g} / \mathrm{dl}, 96,9 \pm 11,8 \mu \mathrm{g} / \mathrm{dl}$, 
$100,3 \pm 14,4 \mu \mathrm{g} / \mathrm{dl}$ y 99,6 $\pm 13,2 \mu \mathrm{g} / \mathrm{dl}$, respectivamente. Los porcentajes de vacas del rebaño con valores plasmáticos de $\mathrm{Zn}$ marginales o carentes por año de muestreo se presentan en la Figura 3. Los resultados obtenidos en los diferentes municipios se presentan en la Tabla 2.

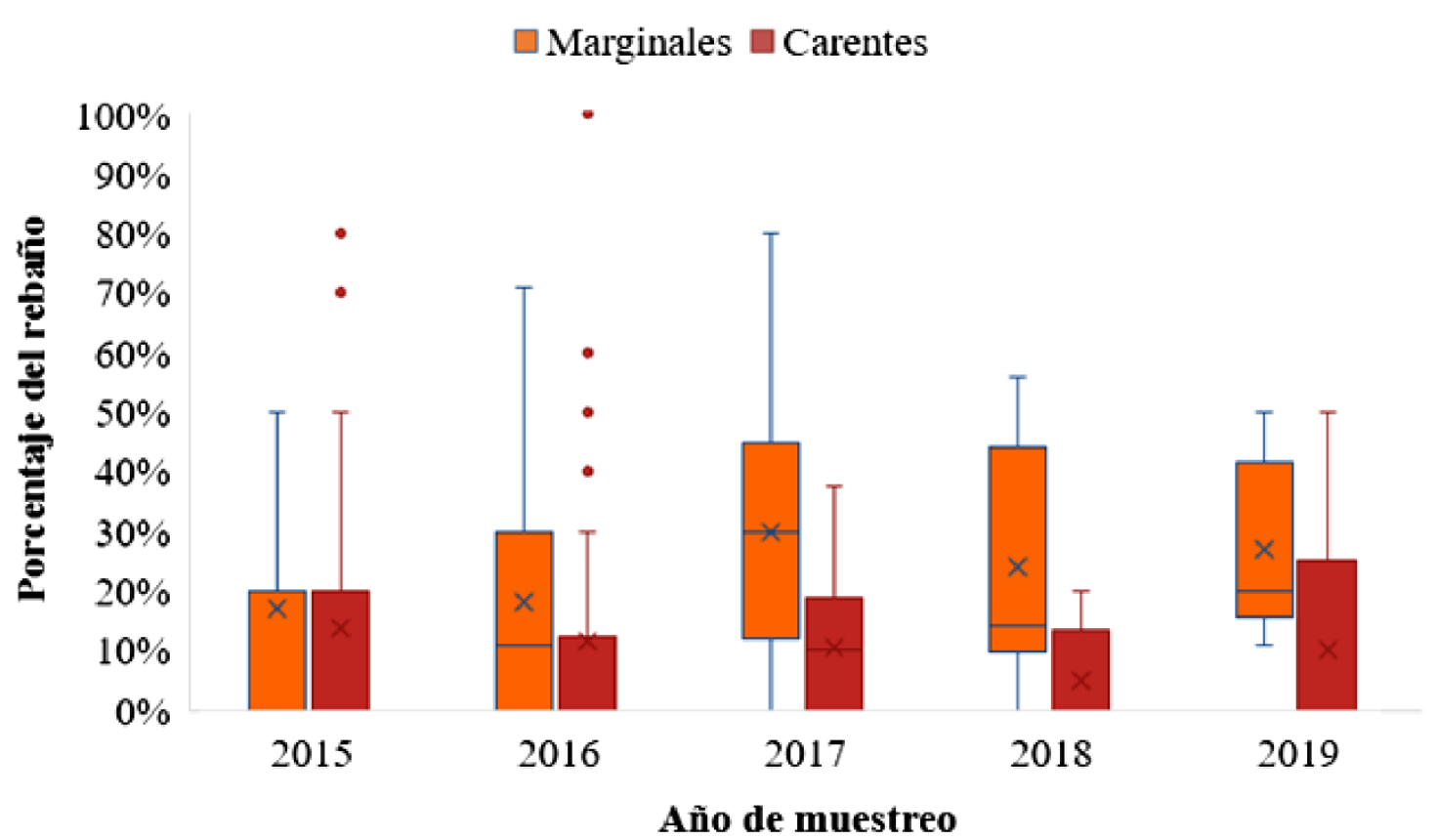

Figura 3. Porcentaje de vacas del rebaño con valores plasmáticos de $\mathrm{Zn}$ marginales (8o a $89 \mu \mathrm{g} / \mathrm{dl}$ ) o carentes $(<80 \mu \mathrm{g} / \mathrm{dl})$ por año de muestreo, en la cuenca deprimida del río Salado (Buenos Aires, Argentina).

\section{Discusión}

En el presente trabajo se determinó que el 31,4 \% de las vacas de cada rebaño presentó valores de concentración de $\mathrm{Zn}$ plasmático marginales o carentes, es decir, por debajo de los valores considerados adecuados. Por lo tanto, este hallazgo apoya la hipótesis planteada, confirmando que existe carencia de $\mathrm{Zn}$ en vacas de la CDRS.

La aparente variación en las concentraciones plasmáticas de $\mathrm{Zn}$ en las distintas estaciones del año podría reflejar cambios estacionales en las concentraciones de $\mathrm{Zn}$ en el forraje. En este sentido, se han comunicado variaciones estacionales en la concentración de $\mathrm{Zn}$ en forrajes en diferentes países, aunque lamentablemente no coinciden entre sí. En Argentina, en la provincia de Formosa, Bernardis et al. (2005) informaron que las concentraciones de $\mathrm{Zn}$ en pastizales en otoño fueron mayores que en verano. En Uruguay, con un sistema de cría similar a la CDRS, Pittaluga Rossi (2018) informó que la concentración de $\mathrm{Zn}$ en los pastizales fue mayor en invierno y primavera, mientras que Ungerfeld (1998), en el mismo país, registró concentraciones mayores de $\mathrm{Zn}$ en el período otoño-invernal. En Noruega, Brekken \& Steinnes (2004) observaron que algunas especies forrajeras contenían mayores concentraciones de $\mathrm{Zn}$ en primavera. Si bien no hay un patrón claro de variación según las estaciones climáticas, cambios en las concentraciones de $\mathrm{Zn}$ en el forraje a lo largo del año podrían explicar, al 
menos en parte, las variaciones observadas en las concentraciones plasmáticas de $\mathrm{Zn}$ en las vacas de la CDRS. Por otro lado, los requerimientos de minerales varían en función del estado fisiológico en el que se encuentran las vacas (Mattioli, 2019). En el invierno, se observaron rebaños en los que un 47,7 \% de vacas presentó valores de concentración plasmática de $\mathrm{Zn}$ marginales o carentes, mientras que en otoño ese valor fue de $21,3 \%$. El invierno, es la época de pariciones en la región, ya que entre el 50 y el $80 \%$ de los rebaños (dependiendo el grado de tecnificación) realizan el servicio durante la primavera (Gómez, 2015; Faverin \& Machado, 2019; Maresca et al., 2011). Esto implica que durante el invierno las vacas se encuentran finalizando su gestación o en lactancia temprana. Los resultados hallados en el presente trabajo podrían deberse al aumento en la demanda de $\mathrm{Zn}$ por parte del feto al final de la gestación (Masters \& Somers, 1980) y a un aumento en la excreción a causa de la lactancia, ya que la leche posee una elevada concentración de $\mathrm{Zn}$ (3 al $5 \mathrm{mg}$ de $\mathrm{Zn} / \mathrm{L}$ ) (Suttle, 2010). Durante el otoño, generalmente las vacas se encuentran promediando su gestación y sin amamantar, ya que habitualmente el destete de los terneros se realiza en esta época del año (Carrillo, 2007; Faverin \& Machado 2019). Esto podría explicar, al menos en parte, el menor porcentaje de vacas con valores de $\mathrm{Zn}$ plasmático marginales o carentes, en los rebaños en el otoño con respecto al invierno.

\begin{tabular}{|c|c|c|c|c|}
\hline Municipio & $\begin{array}{c}\mathrm{Zn} \\
\text { plasmático } \\
\mu \mathrm{g} / \mathrm{dl} \pm \mathrm{DE}\end{array}$ & $\begin{array}{c}\text { Adecuado } \\
\% \pm D E\end{array}$ & $\begin{array}{c}\text { Marginal } \\
\% \pm \mathrm{DE}\end{array}$ & $\begin{array}{c}\text { Carente } \\
\% \pm D E\end{array}$ \\
\hline Ayacucho & $106 \pm 18$ & $66 \pm 36$ & $21 \pm 23$ & $13 \pm 20$ \\
\hline Azul & $109 \pm 6$ & $90 \pm 0$ & $10 \pm 0$ & $0 \pm 0$ \\
\hline Castelli & $106 \pm 13$ & $85 \pm 6$ & $2 \pm 5$ & $13 \pm 10$ \\
\hline Chascomús & $99 \pm 12$ & $60 \pm 22$ & $33 \pm 16$ & $7 \pm 10$ \\
\hline Dolores & $104 \pm 28$ & $20 \pm 28$ & $20 \pm 28$ & $60 \pm 57$ \\
\hline General Alvear & $112 \pm 27$ & $70 \pm 44$ & $14 \pm 17$ & $16 \pm 31$ \\
\hline General Belgrano & $115 \pm 15$ & $54 \pm 36$ & $46 \pm 36$ & $0 \pm 0$ \\
\hline General Lavalle & $108 \pm 12$ & $83 \pm 16$ & $15 \pm 15$ & $2 \pm 6$ \\
\hline General Madariaga & $101 \pm 14$ & $71 \pm 30$ & $22 \pm 18$ & $7 \pm 15$ \\
\hline General Paz & $94 \pm 6$ & $60 \pm 14$ & $35 \pm 7$ & $5 \pm 7$ \\
\hline Las Flores & $115 \pm 30$ & $80 \pm 28$ & $15 \pm 21$ & $5 \pm 7$ \\
\hline Lezama & $93 \pm 16$ & $71 \pm 41$ & $16 \pm 19$ & $13 \pm 22$ \\
\hline Lobos & $98 \pm 15$ & $65 \pm 21$ & $25 \pm 7$ & $10 \pm 14$ \\
\hline Magdalena & $96 \pm 12$ & $60 \pm 28$ & $30 \pm 14$ & $10 \pm 14$ \\
\hline Maipú & $109 \pm 16$ & $73 \pm 29$ & $19 \pm 21$ & $8 \pm 13$ \\
\hline Pila & $93 \pm 6$ & $56 \pm 34$ & $40 \pm 35$ & $4 \pm 7$ \\
\hline Punta Indio & $98 \pm 21$ & $64 \pm 33$ & $21 \pm 16$ & $15 \pm 25$ \\
\hline Rauch & $98 \pm 18$ & $64 \pm 30$ & $20 \pm 19$ & $16 \pm 25$ \\
\hline Saladillo & $102 \pm 13$ & $72 \pm 29$ & $20 \pm 14$ & $8 \pm 15$ \\
\hline Tapalqué & $107 \pm 12$ & $76 \pm 32$ & $12 \pm 8$ & $12 \pm 27$ \\
\hline
\end{tabular}

Tabla 2. Media \pm desvío estándar de las concentraciones plasmáticas de zinc de los rebaños y del porcentaje de vacas dentro de cada uno, con concentraciones plasmáticas de $\mathrm{Zn}$ adecuadas ( $\geq 90 \mu \mathrm{g} / \mathrm{dl}$ ), marginales ( $\geq 80$ y $<90 \mu \mathrm{g} / \mathrm{dl}$ ) y carentes ( $<80 \mu \mathrm{g} / \mathrm{dl}$ ), por municipio. DE: desvío estándar. 
Existen antecedentes en el país de relevamientos realizados con el fin de evaluar el estatus de $\mathrm{Zn}$ en rodeos bovinos. En el municipio de Magdalena (el cual forma parte de la CDRS), Ramírez et al. (1998) realizaron un relevamiento de 13 rebaños en los que evaluaron bovinos mayores y menores a 2 años, en otoño y primavera, y observaron que durante el otoño hubo un mayor porcentaje de animales con valores de $\mathrm{Zn}$ en plasma menores a 80 $\mu \mathrm{g} / \mathrm{dl}$. En el municipio de General Villegas (noroeste de la provincia de Buenos Aires) se registraron valores de $\mathrm{Zn}$ en plasma más elevados durante el invierno. En este caso, la alimentación de las vacas consistió en una base forrajera de pasturas implantadas y verdeos, mientras que, en otro rebaño, cuya fuente forrajera principal fue el pastizal natural, las vacas no presentaron variación estacional (Buffarini et al., 2008). También en General Villegas, Minatel et al. (2004) relevaron las concentraciones de $\mathrm{Zn}$ en plasma en novillos, terneros y vaquillonas de 10 rebaños ganaderos en invierno y verano, observando que, para estas categorías, el valor medio en invierno fue menor al observado en verano (68,5 y 80,5 $\mu \mathrm{g} / \mathrm{dl}$, respectivamente). En el municipio de Balcarce (sudeste de la provincia de Buenos Aires), Cseh et al. (1998) reportaron que tanto vacas como terneras, en el mes de junio presentaron valores plasmáticos de $\mathrm{Zn}$ más altos que en octubre y diciembre. En el municipio de Guaminí (centro oeste de la provincia de Buenos Aires), Coria et al. (2013) realizaron un seguimiento estacional de la concentración plasmática de $\mathrm{Zn}$ de vacas en un rebaño durante dos años (2011 y 2012), sin evidencias de diferencias entre estaciones. En todos los muestreos los valores medios de $\mathrm{Zn}$ en plasma se encontraron entre 60 y $70 \mu \mathrm{g} / \mathrm{dl}$. En la localidad de Coronel Cornejo (norte de la provincia de Salta), Micheloud et al. (2017) evaluaron las concentraciones de $\mathrm{Zn}$ en plasma en vacas y terneras de reposición en las cuatro estaciones del año, sin evidenciar diferencias por estación y con valores medios por encima de $90 \mu \mathrm{g} / \mathrm{dl}$ en las cuatro estaciones, en las dos categorías. Balbuena et al. (1989), en un estudio realizado en vacas en lactancia y terneros en crecimiento (entre 1 y 2 años de edad) en 11 establecimientos situados en el este de las provincias de Formosa y Chaco, reportaron que el $30 \%$ de las muestras presentaban valores de $\mathrm{Zn}$ plasmático por debajo de $80 \mu \mathrm{g} / \mathrm{dl}$, sin observar diferencias entre categorías. Al evaluar estos antecedentes en conjunto, resulta evidente que su variabilidad impide hacer generalizaciones con respecto al comportamiento de las concentraciones plasmáticas de Zn en rodeos de cría. Por ende, para conocer el estatus de $\mathrm{Zn}$ de los rodeos, parecería adecuado relevar cada zona destinada a la cría bovina de manera particular.

El hecho de que en los rodeos se observara un 31,4\% de las vacas con valores de $\mathrm{Zn}$ plasmático por debajo de lo considerado adecuado, indica que existe el riesgo de disminución en la eficiencia productiva y reproductiva de la ganadería en la CDRS. La carencia de $\mathrm{Zn}$ en el bovino genera menores ganancias diarias de peso, asociadas a una reducción en el apetito y a una menor eficiencia de conversión (Alsaad et al., 2006; Engle et al., 1997; Suttle, 2010). Si bien poco se sabe acerca del efecto de la carencia de $\mathrm{Zn}$ en la reproducción de la hembra bovina, se la ha vinculado a disminución de la fertilidad, interrupción de la gestación y retención placentaria (Enjalbert et al., 2006). En terneros machos, por otro lado, esta carencia produce un menor desarrollo testicular (Miller \& Miller, 1962; Pitts et al., 1966). Por último, los 
animales carentes de Zn son más susceptibles a enfermarse debido a fallas de la respuesta inmune celular y humoral, alteraciones en la respuesta inflamatoria y daño de la defensa antioxidante (Bonaventura et al., 2015; Engle et al., 1997; Ibs \& Rink, 2003; Mattioli, 2019). El conocimiento del estatus de Zn en vacas de cría de la CDRS, permitirá optimizar su manejo nutricional y/o sanitario para evitar potenciales pérdidas productivas $\mathrm{y}$ reproductivas.

\section{Conclusión}

Los valores de Zn plasmático por debajo de los considerados adecuados alertan sobre el potencial riesgo de menor eficiencia productiva y reproductiva de las vacas en la CDRS. Más estudios son necesarios para caracterizar y cuantificar las posibles pérdidas por mencionada carencia.

\section{Declaración de conflicto de intereses}

Los autores declaran que no existe conflicto de intereses, incluyendo las relaciones financieras, personales o de otro tipo con otras personas $u$ organizaciones que pudieran influir de manera inapropiada en el presente trabajo.

\section{Agradecimientos}

El presente trabajo se financió con fondos del Servicio de Nutrición Mineral y el Servicio de Asistencia Clínica Poblacional, ambos de la FCV-UNLP. Agradecemos a todos los médicos veterinarios que colaboraron con la toma de muestras, a los laboratorios Regional Rauch, Bazkos Viejos de General Madariaga y CSG Laboratorio de Diagnóstico Veterinario de Lezama, como también al personal de la Estación Experimental Agropecuaria cuenca del Salado del INTA que participó activamente para generar el vínculo con los profesionales y laboratorios de la región. La participación de todos ellos fue muy importante para que se pudiese llevar a cabo este estudio.

\section{Referencias}

Alsaad KM, Al-Sadi HI, Abdul-Majeed MO. 2006. Clinical and pathological studies on naturally occurring zinc deficiency (hypozincemia) in buffalo calves. Proceedings of the 4th Scientific Conference, College of Veterinary Medicine, University of Mosul, Mosul, Iraq, 1:97-107.

Andreini C, Banci L, Bertini I, Rosato A. 2006. Counting the zinc-proteins encoded in the human genome. Journal of Proteome Research. 5(1): 196-201.

https://doi.org/10.1021/pro50361j

Andreini C, Bertini I. 2012. A bioinformatics view of zinc enzymes. Journal of Inorganic Biochemistry. 111:150-6. https://doi.org/10.1016/j.jinorgbio.2011.11.020

Balbuena O, McDowell LR, Toledo HO, Conrad JH, Wilkinson N, Martín FG. 1989. Estudio de la nutrición mineral de los bovinos para carne del este de las provincias de Chaco y Formosa (Argentina). Revista Veterinaria Argentina. 6:584-94. 
Bernardis AC, Roig CA, Bennasar Vilches M. 2005. Productividad y calidad de los pajonales de Sorghastrum setosum (Griseb.) Hitchc. en Formosa, Argentina. Agricultura Técnica. 65(2):177-85. https://doi.org/10.4067/So365-28072005000200007

Bonaventura P, Benedetti G, Albarède F, Miossec P. 2015. Zinc and its role in immunity and inflammation. Autoimmunity Reviews. 14(4):277-85.

Brekken A, Steinnes E. 2004. Seasonal concentrations of cadmium and zinc in native pasture plants: consequences for grazing animals. Science of the Total Environment. 326(13):181-95. https://doi.org/10.1016/j.scitotenv. 2003.11.023

Buffarini MA, Minatel L, Cseh S. 2008. Variaciones estacionales de minerales en sangre en dos rodeos de cría en el partido de General Villegas. Memoria Técnica, INTA. 88-92.

Carrillo J. 2007. Manejo de un rodeo de cría. Décima Reimpresión. Buenos Aires, Editorial Hemisferio Sur SA. Coria ML, Cseh SB, Fernández EL. 2013. Diagnóstico de deficiencias minerales en un campo del partido de Guaminí, Buenos Aires. Revista Argentina de Producción Animal. 33(1):25.

Cseh S, Crenovich H, Fay P, Olmos G, Drake M. 1997. Valor nutritivo de pasturas en campos del este de la Provincia de Buenos Aires, Argentina. XXV Jornadas Uruguayas de Buiatría. IX Congreso Latinoamericano de Buiatría. Paysandú, Uruguay. 29-30.

Cseh S, Ridao M, San Martino S, Drake M, Yarrar M. 1998. Valores serológicos de hierro y zinc en distintas categorías de bovinos hembra. Veterinaria México. 29(1): 23-7.

Engle TE, Nockels CF, Kimberling CV, Weaber DL, Johnson AB. 1997. Zinc repletion with organic or inorganic forms of zinc and protein turnover in marginally zinc-deficient calves. Journal of Animal Science. 75(11):3074-81.

https://doi.org/10.2527/1997.75113074X

Enjalbert F, Lebreton P, Salat O. 2006. Effects of copper, zinc and selenium status on performance and health in commercial dairy and beef herds: retrospective study. Journal of Animal Physiology and Animal Nutrition. 90(11-12):459-66.

https://doi.org/10.1111/j.1439-0396.2006.00627.x

Fallah A, Mohammad-Hasani A, Hosseinzadeh Colagar A. 2018. Zinc is an essential element for male fertility: a review of $\mathrm{Zn}$ roles in men's health, germination, sperm quality, and fertilization. Journal of Reproduction and Infertility. 19(2): 69-81.

Faverin C, Machado C. 2019. Tipologías y caracterización de sistemas de cría bovina de la pampa deprimida. Chilean Journal of Agricultural \& Animal Sciences. 35(1):3-13. http://doi.org/10.4067/So719-38902019005000101

Gómez, L. 2015. Adopción de tecnología de la cría de carne vacuna en la cuenca del Salado. Trabajo Final de Ingeniería en Producción Agropecuaria, Facultad de Ciencias Agrarias, Universidad Católica Argentina. https://reposito rio.uca.edu.ar/handle/123456789/463

Herdt TH, Hoff B. 2011. The use of blood analysis to evaluate trace mineral status in ruminant livestock. Veterinary Clinics of North America: Food Animal Practice. 27(2):255-83. https://doi.org/10.1016/j.cvfa.2011.02.004

Ibs KH, Rink L. 2003. Zinc-altered immune function. Journal of Nutrition. 133(5):1452S-6S. https://doi.org/10.1 093/jn/133.5.1452S

Kambe T, Tsuji T, Hashimoto A, Itsumura N. 2015. The physiological, biochemical, and molecular roles of zinc transporters in zinc homeostasis and metabolism. Physiological Reviews. 95(3):749-84. https://doi.org/10.115 2/physrev.00035.2014

Kincaid R. 1999. Assessment of trace mineral status of ruminants: a review. Journal of Animal Science. 77(1):1-10. https://doi.org/10.2527/jas2000.77e-suppl1x

Lee SR. 2018. Critical role of zinc as either an antioxidant or a prooxidant in cellular systems. Oxidative Medicine and Cellular Longevity. 1-11. https://doi.org/10.1155/2018/9156285

Levaot N, Hershfinkel M. 2018. How cellular Zn2+ signaling drives physiological functions. Cell Calcium. 75:53-63. https://doi.org/10.1016/j.ceca.2018.08.004

Lindskog S. 1997. Structure and mechanism of carbonic anhydrase. Pharmacology and Therapeutics. 74(1):1-20. https://doi.org/10.1016/So163-7258(96)00198-2

Maresca S, Quiróz García JL, Plorutti F. 2011. Eficiencia reproductiva en rodeos de cría de la cuenca del Salado. Buenos Aires, Publicaciones Regionales INTA. 
Masters DG, Somers M. 1980. Zinc status of grazing sheep: seasonal changes in zinc concentration in plasma, wool and pasture. Australian Journal of Experimental Agriculture and Animal Husbandry. 20(102):20-4. https://d oi.org/10.1071/EA9800020

Mattioli GA. 1998. Caracterización de la hipocuprosis bovina en el Partido de Magdalena (Provincia de Buenos Aires). Tesis de Doctorado en Ciencias Veterinarias, Facultad de Ciencias Veterinarias, Universidad Nacional de La Plata.

Mattioli GA. 2019. Nutrición mineral y vitamínica de bovinos. 2da Edición. La Plata, CCB Academic Press.

Micheloud JF, Suárez VH, Martínez GM, Rosa DE, Ventura MB, Mattioli GA. 2017. Niveles séricos de minerales en hembras de cría bovina en un establecimiento de la región de selva y pastizal pedemontano del norte de Salta. Revista FAVE. 16:97-100. https://doi.org/10.14409/favecv.v16i2.7027

Miller JK, Miller WJ. 1962. Experimental zinc deficiency and recovery of calves. Journal of Nutrition. 76:467-74. https://doi.org/10.1093/jn/76.4.467

Minatel L, Buffarini MA, Scarlata EF, Dallorso ME, Carfagnini JC. 2004. Niveles de cobre, hierro, zinc y selenio de bovinos del noroeste de la provincia de Buenos Aires. Revista Argentina de Producción Animal. 24(3-4):225-35.

Ministerio de Agroindustria, Presidencia de la Nación, Subsecretaría de Ganadería. 2015. Caracterización de la producción bovina. Sistema de monitoreo del sector de la carne bovina. [En línea] Disponible en:

https://inta.gob.ar/sites/default/files/inta_caracterizacion_de_la_produccion_bovina.p df.

Némoz JP, Giancola SI, Bruno MS, de la Vega MB, Calvo S, Di Giano S, Rabaglio MD. 2013. Causas que afectan la adopción de tecnología en la ganadería bovina para carne de la Cuenca del Salado: enfoque cualitativo. Estudios socioeconómicos de la adopción de tecnología. $\mathrm{N}^{\mathrm{o}}$ 5. INTA.

Nicholas DJD. 1975. Trace elements in soil-plant-animal systems. Glen Osmond, Australia, Academic Press. National Research Council (NRC). 2016. Nutrient requirements of beef cattle. Eighth revised edition: update 2016. Washington, DC, The National Academies Press.

Olechnowicz J, Tinkov A, Skalny A, Suliburska J. 2018. Zinc status is associated with inflammation, oxidative stress, lipid, and glucose metabolism. Journal of Physiological Sciences. 68(1):19-31. https://doi.org/10.1007/s1257 6-017-0571-7

Otondo J. 2011. Efectos de la introducción de especies megatérmicas sobre características agronómicas y edáficas de un ambiente halomórfico de la Pampa Inundable. Tesis de Maestría, Facultad de Agronomía, Universidad de Buenos Aires.

http://ri.agro.uba.ar/files/download/tesis/maestria/2011otondoJose.pdf

Pittaluga Rossi AM. 2018. Minerales en campo natural: variación estacional y por sitio geográfico del contenido de fósforo, cobre, manganeso, zinc, hierro y selenio. Tesis de Maestría, Facultad de Ciencias y Facultad de Agronomía, Universidad de la República, Uruguay. https://hdl.handle.net/20.500.12008/21453

Pitts WJ, Miller WJ, Fosgate OT, Morton JD, Clifton CM. 1966. Effect of zinc deficiency and restricted feeding from two to five months of age on reproduction in Holstein bulls. Journal of Dairy Science. 49(8):995-1000.

https://doi.org/10.3168/jds.Soo22-0302(66)87997-3

Ramírez CE, Mattioli GA, Giuliodori MJ, Yano H, Matsui I. 1998. Deficiencia de Zn en bovinos de cría de la provincia de Buenos Aires. Veterinaria Argentina. 15(142):114-8.

Robson AD. 1993. Zinc in soils and plants. Proceedings of the International Symposium on 'Zinc in Soils and Plants'. University of Western Australia, Perth, Australia, p. 59-71.

Rojas MC, Vázquez PM, Verdier M, Noseda R. 2011. Componentes del paisaje que favorecen la aparición de carbunco en la Pampa Deprimida (provincia de Buenos Aires, Argentina). Revue scientifique et technique (International Office of Epizootics). 30(3):897-909. https://doi.org/10.20506/rst.30.3.2086 
Rossanigo CE, Arano A, Rodríguez Vázquez G. 2012. Stock 2012 del ganado bovino. Mapas de existencias e indicadores ganaderos. Información Técnica $\mathrm{N}^{\circ}$ 187. EEA INTA San Luis.

SENASA. 2015. Indicadores de ganadería bovina. [En línea] Disponible en: http://www.senasa.gob.ar/prensa/DNSA/Control_Gestion_y_Programas_Especiales/In dicadores_ganaderos/1_Indicadores_Ganaderia_Bovina/Gan aderia_Bovina.html.

Suttle NF. 2010. Mineral nutrition of livestock. 4th Ed. Oxfordshire, Reino Unido, CAB International.

Taneja SK, Kaur R. 1990. Pathology of ovary, uterus, vagina and gonadotrophs of female mice fed on Zn-deficient diet. Indian Journal of Experimental Biology. 28(11):1058-65.

Tapiero H, Tew KD. 2003. Trace elements in human physiology and pathology: zinc and metallothioneins. Biomedicine and Pharmacotherapy. 57(9):399-411.

https://doi.org/10.1016/So753-3322(03)00081-7

Tyler G, Olsson T. 2001. Plant uptake of major and minor mineral elements as influenced by soil acidity and liming. Plant and Soil. 230:307-21.

https://doi.org/10.1023/A:1010314400976

Ungerfeld E. 1998. Factores que afectan el contenido de minerales en pasturas naturales y el estado nutricional de vacunos y ovinos en Uruguay. INIA Tacuarembó - Revisión Minerales. p. 184-208.

Vallee BL, Falchuk KH. 1993. The biochemical basis of zinc physiology. Physiological Reviews. 73(1):79-118. https://doi.org/10.1152/physrev.1993.73.1.79

Vázquez P, Rojas MC, Burges JC. 2008. Caracterización y tendencias de la ganadería bovina en la cuenca del Salado. Veterinaria Argentina. 25(248):572-84. 